\title{
Health-care providers' preparedness for H1N1/09 influenza prevention and treatment in Dar es Salaam, Tanzania
}

\author{
Appolinary Kamuhabwa and Reena Chavda
}

Unit of Pharmacology and Therapeutics, School of Pharmacy, Muhimbili University of Health and Allied Sciences, Dar es Salaam, Tanzania

\begin{abstract}
Introduction: In Tanzania, the first case of H1N1/09 influenza was reported in September 2009. By March 2010, the reported number of cases was 770 with one death. Due to shortage of qualified human resources, essential medicines and laboratory supplies in health facilities, it was not known how well health-care providers in the country were prepared to deal with the pandemic.

Methodology: The study was conducted from December 2009 to May 2010 in public hospitals and private community pharmacies in Dar es Salaam, Tanzania. Fifty-three prescribers and 200 dispensers were interviewed to assess their knowledge on the prevention and treatment of H1N1/09 influenza, and dissemination of correct information to the public regarding the pandemic.

Results: Most participants had inadequate knowledge on the transmission, prevention and management of H1N1/09 influenza. The majority of prescribers knew neither the antiviral drugs recommended for the treatment of H1N1 influenza (64\%) nor their adverse effects (92.5\%). The drug dispensers' knowledge was also inadequate regarding the transmission, prevention and treatment of H1N1/09 influenza. Forty-one (20.5\%) salespersons without formal training in pharmaceutical or medical sciences were found dispensing and supervising the pharmacies. Most participants had misconceptions on how H1N1/09 virus was transmitted.

Conclusion: The results of the study revealed gaps in the knowledge of the health-care providers on the prevention and treatment of H1N1/09 influenza infection. This indicates the need for strengthening of health-care systems and provision of continuing education and professional development for health-care providers on new and re-emerging diseases in the community.
\end{abstract}

Key words: dispensers; H1N1/09 flu; influenza; hospitals; knowledge; pandemic,; prescribers; pharmacies

J Infect Dev Ctries 2012; 6(3):262-270.

(Received 06 October 2010 - Accepted 11 October 2011)

Copyright (C) 2012 Kamuhabwa and Chavda. This is an open-access article distributed under the Creative Commons Attribution License, which permits unrestricted use, distribution, and reproduction in any medium, provided the original work is properly cited

\section{Introduction}

Even a mild pandemic may result in a great loss of life. Therefore, an epidemic in a neighboring country should be enough cause for government and health-care systems to be alerted and get well prepared to contain the epidemic, should it spread across the border into the country. In Tanzania, the first case of H1N1/09 influenza was reported in September 2009. By March 2010, there were already 770 such cases and one death [1]. In a number of regions and districts in the country, reports were coming in of people being infected with the virus. The most affected area, with 384 confirmed cases, was Mbulu district in Manyara region, in the north of the country. In Dar es Salaam, the commercial capital of Tanzania, 211 cases were confirmed, with the most recent detected through the Routine Surveillance of Influenza, reported on 17 March 2010 [2].

Following an epidemiological update on the status of H1N1/2009 influenza around the world, the
World Health Organization (WHO) declared the end of the pandemic on 10 August 2010. The WHO predicts that, in the post-pandemic period, cases and outbreaks of H1N1 virus will continue to occur. It is therefore recommended that people continue to stay alert and protect themselves from the infection through use of vaccines, practicing hand and respiratory hygiene, and other necessary measures.

In dealing with disease outbreaks, including that of H1N1/2009, Tanzania faces a number of challenges. Public health facilities often lack adequate trained manpower, facilities, drugs and laboratory supplies. Despite the growing number of private health facilities (particularly in urban areas), shortage of human resources for health remains a major problem. Currently, there are an estimated 5,513 health facilities operating in the country. These include referral, regional and district hospitals, health centers, and dispensaries. According to the proposed staffing levels, a total of 125,924 health workers are 
Table 1. Questions on knowledge of prescribers regarding H1N1/09 influenza

\begin{tabular}{|l|c|c|c|c|}
\hline \multirow{2}{*}{ Hospital } & \multicolumn{4}{|c|}{ Number of prescribers per department in each hospital } \\
\cline { 2 - 5 } & OPD & Antenetal Clinic & HIV Clinic & Paediatrics Clinic \\
\hline Mwananyamala & 3 & 2 & 3 & 2 \\
\hline Temeke & 3 & 2 & 2 & 2 \\
\hline Amana & 2 & 2 & 7 & $\mathbf{1 5}$ \\
\hline Muhimbili & 9 & 4 & $\mathbf{1 0}$ & $\mathbf{1 1}$ \\
\hline TOTAL & $\mathbf{1 7}$ & & & 2 \\
\hline
\end{tabular}

required to man these facilities. The actual number of currently available professional health-care workers is 35,202 . This gives a deficit of $72 \%$ for both public and private health facilities [3]. A number of factors have contributed to the poor health and social welfare situation as a result of the human resource crisis in the country. These include the Retrenchment Policy, the employment freeze implemented between 1993 and 1999 to downsize the workforce, poor planning and forecasting of human resource requirements, and the emergence of HIV and AIDS [3].

Being close to homes and thus easily accessible, community pharmacies play an important role as a source of drugs and drug information [4,5]. In Tanzania, community pharmacies complement public health facilities by providing these services, which are otherwise unavailable in the public facilities [6]. Poor quality care, shortage of skilled health-care providers, frequent essential medicine stock-outs and long waiting times in the health-care facilities are the main reasons people seek and buy drugs from community pharmacies [7]. It would therefore be expected that, during the outbreak of H1N1/09 influenza in Tanzania, many people may have visited the pharmacies seeking treatment and medical advice. To provide accurate information, drug dispensing personnel in the pharmacies should be well equipped with the knowledge on the transmission, prevention and treatment of H1N1/09 influenza.

Lack of knowledge among health-care providers on the transmission, prevention and treatment of the influenza may contribute to the disease being widely spread in the country. Although H1N1/09 influenza is recognized as a public health, social, economic and development threat, no study has been conducted in the country to assess health personnel preparedness in terms of awareness and knowledge on the transmission, prevention and treatment of the disease. It is thus not known how well these workers are prepared for the management of H1N1/09 influenza and how well versed they are in the provision of accurate information on the same. This study was therefore performed to assess the knowledge of prescribers working in the public hospitals and drug dispensers working in private pharmacies on the transmission, prevention and management of H1N1/09 influenza.

\section{Methodology}

Study area and sample size

This cross-sectional study was conducted in Dar es Salaam, Tanzania, in three public municipal hospitals of Amana, Mwananyamala and Temeke, the referral Muhimbili National Hospital, and private community pharmacies.

A total of 53 prescribers were interviewed from the hospitals. From each of the 200 private pharmacies selected for the study, one drug dispenser was interviewed. Two laboratory staff members working at the National Institute for Medical Research (NIMR) laboratory of the Ministry of Health and Social Welfare (MoHSW) were also interviewed. Thus the study involved 53 prescribers, 200 dispensers, and two laboratory personnel.

\section{Prescribers}

The prescribers were selected from the Outpatient Department (OPD), Antenatal Clinic, Pediatric Clinic and HIV Clinic (Table 1) based on the fact that patients attending these clinics are highly susceptible to HIN1 influenza infection $[8,9]$. 
Table 2. Socio-demographic characteristics

\begin{tabular}{|l|c|c|c|}
\hline \multirow{2}{*}{ Categories of Drug Dispensers } & \multicolumn{3}{|c|}{ Number of Drug Dispensers per Municipal } \\
\cline { 2 - 4 } & Temeke & \multicolumn{2}{|c|}{ Ilala } \\
\hline Pharmacists & 9 & 22 & 31 \\
\hline Nurse assistants & 3 & 9 & 4 \\
\hline Pharmaceutical Technician & 7 & 35 & 39 \\
\hline Sales person & 4 & 19 & $\mathbf{8 5}$ \\
\hline TOTAL & $\mathbf{2 3}$ & $\mathbf{9 2}$ \\
\hline
\end{tabular}

\section{Drug dispensing personnel}

In each of the visited 200 private community pharmacies, one drug dispenser was interviewed. Selection of the pharmacies was done on a random sampling technique. A list of currently registered community pharmacies in Dar es Salaam was obtained from the Tanzania Food and Drugs Authority (TFDA). By December 2009, there were a total of 233 community pharmacies registered in Dar es Salaam (27 in Temeke, 96 in Ilala, and 110 in Kinondoni municipalities). The number of pharmacies visited in each municipality depended on the number of pharmacies present in the individual municipality (Table 2).

The pharmacist was the main target of the study; however, if the pharmacist was not available, the interviews were conducted on a pharmaceutical technician, nurse assistant, or any other person found dispensing drugs in the pharmacy.

\section{Knowledge assessment}

Semi-structured questionnaires were used as data collecting tools. Questions were designed to assess the awareness and knowledge of health-care providers on the transmission, prevention, treatment and supportive care of H1N1/09 influenza patients. The main survey questions were based on the cause of H1N1/2009 influenza, transmission of the virus, signs and symptoms of the disease, readiness of health-care workers in providing care for infected patients, management of the patients, preventive measures, antiviral drugs used for the treatment of the disease and their side effects, other treatment measures, and detection of the virus in body fluid samples.

A knowledge scale with equally weighted subquestions was used. One point was given for a correct response and zero for an incorrect answer. In the majority of the questions, interviewees were expected to give a certain number of correct answers. Such questions were scored differently. For instance, if a question required 8 correct answers, the scoring was divided into 4 parts $(0,1-3,4-6,7-8)$. If an interviewee could not give a single correct answer, he/she was regarded as having no knowledge; if between one and three correct answers were given, the interviewee had low knowledge; if between four and six correct answers were given, the interviewee had medium knowledge, and if between seven and eight correct answers were given, the interviewee had high knowledge.

Interviews on personnel at the National Institute for Medical Research Laboratory

Laboratory diagnosis of H1N1/09 virus was not performed in the study hospitals but at the NIMR laboratory. Samples (nasopharyngeal or nasal swabs) collected from suspected/infected patients were being sent to the laboratory at NIMR for analysis and confirmation of the virus. Therefore, two laboratory personnel working in this laboratory were interviewed regarding sample collection, processing, and confirmation of the virus.

\section{Data analysis}

Responses to questions were coded and analyzed using the EPI INFO 3.4 computer software version (Centers for Disease Control and Prevention, Atlanta, GA, USA). Questionnaires contained questions designed to gather information on socio-demographic characteristics and knowledge levels of respondents regarding H1N1/09 influenza. Chi square and Fischer Exact test were used to calculate P-values for comparison of study variables. Results were considered significant if P-value was less than 0.05. 
Table 3. Knowledge levels of prescribers regarding H1N1/09 flu symptoms, supportive care, pharmacological treatment, side effects of medications and preventive measures $(n=53)$

\begin{tabular}{|l|c|c|c|c|}
\hline \multirow{2}{*}{$\begin{array}{l}\text { Questions on Knowledge of Prescribers Regarding } \\
\text { H1N1/09 flu }\end{array}$} & \multicolumn{4}{|c|}{ Knowledge Levels of Prescribers } \\
\cline { 2 - 5 } & None & Low & Medium & High \\
\hline Transmission & $0(0 \%)$ & $1(2 \%)$ & $9(17 \%)$ & $35(66 \%)$ \\
\hline Symptoms & $6(11.3 \%)$ & $3(5.7 \%)$ & $16(30.2 \%)$ & $28(52.8 \%)$ \\
\hline Supportive care & $7(13.2 \%)$ & $14(26.4 \%)$ & $22(41.5 \%)$ & $10(18.9 \%)$ \\
\hline Preventive measures & $9(16.9 \%)$ & $17(32.1 \%)$ & $15(28.3 \%)$ & $12(22.6 \%)$ \\
\hline Antiviral drugs & $34(64.2 \%)$ & $5(9.4 \%)$ & $12(22.6 \%)$ & $2(3.8 \%)$ \\
\hline Antiviral side effects & $49(92.5 \%)$ & $2(3.8 \%)$ & $1(1.9 \%)$ & $1(1.9 \%)$ \\
\hline
\end{tabular}

Permission to conduct the study and ethical clearance Initial permission to conduct the study in the selected hospitals and private community pharmacies was obtained from Municipal Medical In-charges and TFDA respectively. The written permissions were then submitted to medical officers in charge of the hospitals and owners of the pharmacies.

Informed consent was obtained from the study participants. Ethical clearance was granted by the Senate Research and Publications Committee of Muhimbili University of Health and Allied Sciences.

\section{Results}

\section{Prescribers}

A total of 253 study participants, including 53 prescribers (Table 1) and 200 drug dispensers (Table 2) were interviewed. Of the prescribers, $40(75.5 \%)$ were males and $13(24.5 \%)$ were females. Overall, the majority of the prescribers had high knowledge on the transmission $(66 \%)$ and symptoms $(52.8 \%)$ of H1N1/09 influenza. However, the majority of prescribers $(64 \%)$ did not know the antiviral drugs recommended for the treatment of $\mathrm{H} 1 \mathrm{~N} 1$ influenza, nor their adverse effects $(92.5 \%)$.

Even among those who knew that neuraminidase inhibitors (oseltamivir/Tamiflu and Zanamivir/Relenza) were the antiviral drugs indicated for the treatment of H1N1/09 influenza [7,8], the majority $(86.8 \%)$ of them could not give the correct dosage of these drugs.

The results of knowledge levels of prescribers on H1N1/09 influenza are summarized in Table 3. Because of the small number of prescribers, it was not possible to make a quantitative comparison of knowledge among prescribers from the different departments (OPD, antenatal, HIV and pediatrics) of the selected hospitals.
About a third of the prescribers $(34.0 \%)$ believed that the virus could also be transmitted through contact with pigs and eating pork products. About half (52.8\%) of them had high knowledge regarding the symptoms of H1N1/09 influenza; 19 prescribers (35.8\%) had low to medium knowledge, while six $(11.3 \%)$ could be considered to have had no knowledge at all. Only $10(18.9 \%)$ prescribers were highly knowledgeable on the supportive care for patients presenting with $\mathrm{H} 1 \mathrm{~N} 1 / 09$ symptoms, while the majority $(67.9 \%)$ had low to medium knowledge.

On preventive measures taken during the H1N1/09 influenza outbreak, the majority of the prescribers had medium (28.3\%) to low (17.1\%) knowledge. Asked if they were ready to take care of patients with H1N1/09 influenza, 47.2\% were hesitant, giving reasons such as lack of protective gear and the unavailability of antiviral drugs and vaccines.

Results of prescribers' responses regarding measures the government should take during an outbreak such as H1N1/09 influenza are presented in Figure 1. The majority $(86.8 \%)$ of the prescribers advocated educating the public and health-care workers on H1N1/09 influenza. Other measures recommended by the prescribers included provision of assistance $(37.7 \%)$ to infected individuals and their families, isolating infected people $(37.4 \%)$ to curb the spread of the virus, and ensuring adequate availability of antiviral drugs, supportive care and protective gear, including hand sanitizers, disinfectants, gloves and face masks.

On sources of information through which the prescribers learned about H1N1/09 influenza, the majority $(75.5 \%)$ responded that they received information through mass media (television, newspapers and radio), the internet (30.2\%) and information provided at the health facilities (17\%). 
Table 4. Knowledge levels of drug dispensing personnel regarding H1N1/09 flu symptoms, pharmacological treatment, side effects of medication, supportive care, preventive measures and awareness of areas most by the virus in Tanzania $(n=200)$.

\begin{tabular}{|l|c|c|c|c|c|c|c|c|}
\hline \multirow{2}{*}{$\begin{array}{l}\text { Questions on } \\
\text { Knowledge Regarding }\end{array}$ H1N1/09 flu } & \multicolumn{2}{|c|}{$\begin{array}{c}\text { Knowledge Levels of Pharmaceutical } \\
\text { Personnel }\end{array}$} & \multicolumn{2}{|c|}{ Knowledge Levels of Non-pharmaceutical } \\
personnel
\end{tabular}

\section{Drug dispensers}

A total of 200 drug dispensers were interviewed. These included $62(30.9 \%)$ pharmacists, $81(40.8 \%)$ nursing assistants, $16(7.9 \%)$ pharmaceutical technicians, and $41(20.4 \%)$ salespersons. The salespersons were drug dispensing personnel without formal pharmaceutical training (Table 2). Results on knowledge levels of drug dispensers regarding $\mathrm{H} 1 \mathrm{~N} 1$ influenza are presented in Table 4. In general, pharmaceutical personnel were more knowledgeable $(\mathrm{p}<0.05)$ on the symptoms, supportive care, preventive measures and antiviral drugs indicated for H1N1/09 influenza than non-pharmaceutical personnel.

While $32 \%$ of pharmaceutical personnel had low to moderate knowledge, $38.5 \%$ of nonpharmaceutical personnel had no or low knowledge regarding the transmission of $\mathrm{H} 1 \mathrm{~N} 1 / 09$ virus. No drug dispenser was found to have high knowledge, and the majority $(69.7 \%)$ believed that the virus was transmitted through contact with pigs or eating pork products. About a fifth of both pharmaceutical and non-pharmaceutical personnel were highly knowledgeable with regard to the symptoms of the disease. Moreover, 27 (13.5\%) of the nonpharmaceutical personnel could not describe any symptoms of H1N1/09 influenza.

Some of the mentioned supportive care measures for patients with H1N1/09 influenza included control of fever and pain, and giving adequate fluids. Only 20 (9.9\%) drug dispensers had high knowledge regarding supportive care for the patients. The majority (43\%) of the drug dispensers with no or low knowledge had no pharmaceutical background, while $29.5 \%$ of those with medium to high knowledge were pharmaceutical personnel. The results indicated a high correlation between level of training and knowledge $(\mathrm{P}<0.01$.

Out of the 200 drug dispensers, only five $(1.51 \%)$ could be considered highly knowledgeable of the antivirals used for the management of $\mathrm{H} 1 \mathrm{~N} 1$ influenza. A large proportion $(61 \%)$ of the dispensers, the majority $(47 \%)$ of whom were nonpharmaceutical personnel, did not know the antivirals recommended for the treatment of influenza. Moreover, the majority (92\%) did not know the adverse effects associated with the drugs.

To assess the awareness of the dispensers about H1N1/09 influenza, they were also asked if they knew the districts and regions most affected by H1N1/09 influenza in Tanzania, and the reasons for the high prevalence of the disease in those areas. The purpose was to assess their knowledge on factors that may enhance the spread of the virus in the community. In this regard the participants' knowledge was between low (37.5\%) and medium $(48.5 \%)$.

For preventive measures against H1N1/09 influenza, the majority (81\%) of drug dispensers advised that the government should conduct sensitization campaigns and educate the public through mass media. About a third $(32.5 \%)$ of them 
Figure 1. Prescribers' opinion on actions the government should take during an outbreak such as H1N1/09 flu $(\mathrm{n}=53)$

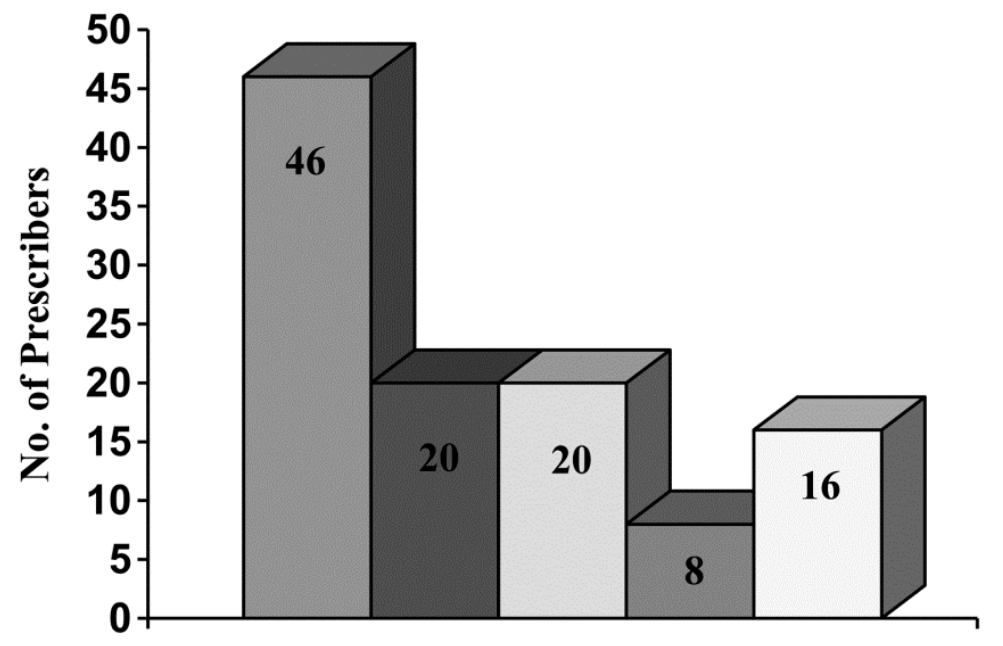

$\square$ Educate citizens
$\square$ Provide Aids
$\square$ Quarantine
$\square$ Ensure availability of
medicines and protective
gears
$\square$ Provide vaccination

were of the view that the most effective way to contain the pandemic was through vaccination. Other measures that they mentioned included provision of adequate supplies of free antiviral drugs and protective gear such as masks, gloves and antiseptics $(44 \%)$, provision of assistance $(44 \%)$ by mobilizing health-care workers into visiting affected communities, and isolating the infected individuals to contain the spread of the virus $(10 \%)$.

With regard to the effective means of disseminating information on disease outbreaks, the dispensers were required to mention the sources through which they received information about H1N1/09 influenza. The majority $(88.2 \%)$ received information through the radio, television and newspapers and others $(25.7 \%)$ from the internet. A small proportion $(9.2 \%)$ responded that they obtained the information through literature and advertisements available at their places of work.

Most $(169 ; 84.3 \%)$ of drug dispensers responded that H1N1/09 influenza was a serious disease and needed special attention by policy makers, health-care providers and the community as a whole. However, the majority (95.4\%) of the dispensers admitted that their lifestyles had not changed despite being aware that Dar es Salaam was one of the regions affected by the H1N1/09 influenza pandemic. The few who reported to have changed their lifestyles said they did so by avoiding contact with pigs and eating pork, wrongly believing that these were the main sources of transmission of the virus.
Responses of NIMR Laboratory personnel on detection of H1N1 virus

The three municipal hospitals (Amana, Mwananyamala and Temeke) and Muhimbili National Hospital that were visited to interview the prescribers did not perform H1N1/09 virus detection tests. The tests were carried out at the NIMR laboratory. To obtain details on how sample collection, processing and detection of the virus were done, two laboratory personnel working at the laboratory were interviewed. The laboratory personnel had undergone a two-week special training on $\mathrm{H} 1 \mathrm{~N} 1 / 09$ virus testing and specimen processing. The training included sample collection, storage, transportation and packing.

The laboratory staff reported facing a number of challenges in conducting their work. Some samples were received in a bad state, mainly due to being subjected to poor transportation and storage conditions from the time they were collected to the time they reached the laboratory. In addition, information in the sample collection forms was poorly recorded.

The laboratory personnel commented that, since some of the samples had not been properly stored during collection and transportation, their viability could not be guaranteed, and therefore the number of confirmed H1N1/09 influenza cases in Tanzania could have been underestimated and understated.

\section{Discussion}

The recent H1N1-09 influenza pandemic emphasized the need for strengthening of health-care systems to contain such cases in the future [12]. In 
response to the pandemic, the Tanzanian government set up medical check-ups at its major entry points, including international airports and borders. By March 2010, 770 H1N1/09 cases and one death [2] had been reported in the country. In June 2009, the WHO provided Tanzania with 51,128 doses of Oseltamivir, an effective drug in the treatment of H1N1 influenza. This survey was therefore conducted at a time when the pandemic was being highly publicized in the country and around the globe. Information about H1N1/09 influenza was available through the media and internet. For effective public education and patient management, health-care providers were expected to have taken the initiative to update themselves with current information about the transmission, symptoms, prevention, and management of H1N1/09 influenza.

To effectively deal with such pandemics, healthcare providers have to be aware and knowledgeable about the disease transmission, symptoms, prevention and treatment. In this study, prescribers working in public hospitals and drug dispensers working in private community pharmacies were found to have inadequate knowledge on the transmission, symptoms, supportive care and preventive measures of H1N1/09 influenza, and the antiviral drugs recommended for the disease. Interestingly, about a third of the prescribers and the majority of the drug dispensers believed that the virus was transmitted through contact with pigs and eating pork products. Results from a similar study conducted in India showed that about half of 791 respondents in the general community believed that H1N1/09 was caused by pigs [13]. Since it is known that public perception or beliefs about an outbreak is influenced by advice and directives by officials and health-care providers [14], it is most likely that the Tanzanian public had not received correct information and advice during the outbreak of H1N1/09 influenza in the country.

Various non-pharmaceutical interventions such as detection, isolation and quarantine are required to contain H1N1/09 influenza. In addition, a sustained supply of respiratory masks and other protective gear such as gloves and antiseptics is one of the activities advocated to combat the spread of the virus in the community. Although the majority of the prescribers and dispensers indicated that H1N1/09 influenza was a serious health problem requiring educating the public on its prevention measures, none of them adopted preventive measures necessary during an air-borne disease outbreak. The lack of substantial change in adopting preventive measures or knowledge about the modes of H1N1 transmission among the health-care providers suggests that community mitigation measures for H1N1/09 influenza and other outbreaks are unlikely to succeed if health-care providers do not take the leading role $[15,16]$.

Vaccination for H1N1/09 influenza is the most effective way of preventing influenza related morbidity and mortality [17]. In this study, only $34.7 \%$ of the prescribers recommended vaccination as a way of containing the virus. Studies elsewhere have shown strong support for H1N1/09 vaccination among health-care providers. For instance, a study done in Canada showed that $52 \%$ of family physicians and $72 \%$ of paediatricians agreed that seasonal influenza vaccines were very useful in the protection of a child's health. In addition, more than $70 \%$ of family physicians and paediatricians considered that the H1N1/09 pandemic vaccine would be well accepted by the public, and more than $80 \%$ thought that it would be well accepted by the vaccinators [18]. In a similar study done in British Columbia in Canada, $69 \%$ of health-care workers reported intending to receive H1N1/09 vaccine [19]. The reasons for most prescribers not recommending vaccination for H1N1/09 influenza in this study may be due to lack of knowledge about the beneficial effects of vaccines and unavailability of these vaccines in the health facilities in Tanzania.

Health facilities in Tanzania are often faced with severe shortages of trained human resources for health, and inadequate drug and laboratory supplies. The current doctor-to-population ratio in Tanzania is about 1:25,000. In addition, the work force continues to experience loss of skilled health workers through attrition [3]. As a result, prescribers in public health facilities experience high patient workload and may therefore have limited time for undergoing continuing education.

In this study, out of the expected 200 pharmacists, only $62(31 \%)$ were available in the private community pharmacies. In addition, 41 (20.5\%) salespersons with no formal training in pharmaceutical or medical sciences were found supervising the pharmacies and dispensing drugs. The lack of knowledge among health-care workers regarding H1N1/09 influenza, observed in this study could partly be due to the lack of adequate and trained human resources for health in Tanzania [20].

Successful recovery of viruses from clinical specimens depends on the quality of material received for inoculation onto cells or eggs [21]. Many viruses are susceptible to drying, adverse $\mathrm{pH}$, and varying osmotic potential. For this reason samples should be 
placed in virus transport media and stored at $4{ }^{\circ} \mathrm{C}$ immediately after being collected at the collection site, and should be inoculated into susceptible cell cultures as soon as possible. If specimens cannot be processed within 48 to 72 hours, they should be kept frozen at or below $-70^{\circ} \mathrm{C}$. Nasopharyngeal and nasal swab samples that were received at the NIMR laboratory were not properly stored during collection and transportation. It is therefore not known whether the improperly stored samples may have resulted in the underestimation of the number of confirmed H1N1/09 virus infection cases during the pandemic in Tanzania.

To our knowledge, this is the first study conducted to assess the knowledge of health-care providers on H1N1/09 influenza in Tanzania. It provides some important information on the preparedness of health-care workers in dealing with disease outbreaks such as N1H1/09 influenza. The findings of this study emphasize the necessity for health-care workers to update themselves with current information on new and re-emerging diseases in the community. To effectively deal with disease outbreaks, it is imperative for the government to strengthen the health-care systems in terms of human personnel as well as medical and laboratory supplies.

The study was conducted only in Dar es Salaam. The number of respondents is relatively too small for a social study. Further studies are recommended to assess the overall knowledge of different cadres of health-care providers and their preparedness in dealing with disease outbreaks in the country.

\section{Acknowledgements}

We wish to express our sincere appreciation to the medical officers in charge of the three municipal hospitals (Amana, Mwananyamala and Temeke) and Muhimbili National Hospital for their invaluable assistance during this study. We thank the Ministry of Health and Social Welfare for their useful guidance throughout the entire period of the study. We are in gratitude to the Tanzania Food and Drugs Authority for providing us with the list of retail private community pharmacies in Dar es Salaam. The laboratory personnel at the NIMR Laboratory are deeply appreciated for their valuable information regarding laboratory detection of H1N1/09 virus. Last, but not the least, our sincere thanks go to all prescribers and dispensers who agreed to spend their precious time to participate in the study.

\section{References}

1. Center for Disease Control and Prevention (CDC) Swine influenza A (H1N1) infection in two children-Southern California, March-April 2009 (2009) MMWR Morb Mortal Weekly Report 58: 400-402.

2. WHO (2010) Global Alert and Response. Pandemic (H1N1) 2009 - update 96. Weekly update; 16 April 2010.

3. Ministry of Health and Social Welfare, United Republic of Tanzania. Human Resources for Health Strategic Plan 2008-
2013. Available at http://www.moh.go.tz. Last accessed 27 February 2012.

4. Kachur SP, Schulden J, Goodman CA, Kassala H, Elling BF, Khatib R, Causer LM, Mkikima S, Abdulla S, Peters B (2004) Prevalence of malaria parasitemia among clients seeking treatment for fever or malaria at drug stores in rural Tanzania 2004. Trop Med Int Health 11: 441-451.

5. Abuya TO, Mutemi W, Karisa B, Ochola SA, Fegan G, and Marsh (2007) Use of over-the-counter malaria medicines in children and adults in three districts in Kenya: Implications for private medicine retailer interventions. Malar J 6: 57.

6. Hetzel MW, Dillip A, Lengeler C, Obrist B, Msechu J, Makemba A, Mshana C, Schulze A, Mshinda H (2008) Malaria treatment in the retail sector: Knowledge and practices of drug sellers in rural Tanzania. BMC Public Health 8: 157.

7. Mamdani M, Bangser M (2004) Poor people's experiences of health services in Tanzania: a literature review. Reprod Health Matters 12: 138-153.

8. Hanshaoworakul W, Simmerman JM, Narueponjirakul U, Sanasuttipun W, Shinde V, Kaewchana S, Areechokechai D, Levy J, Ungchusak K (2009) Severe human influenza infections in Thailand: oseltamivir treatment and risk factors for fatal outcome. PLoS ONE 4: e6051.

9. Vaillant L, La Ruche G, Tarantola A, Barboza P (2009) Epidemic Intelligence Team at InVS Epidemiology of fatal cases associated with pandemic H1N1 influenza. Euro Surveill 14: pii: 19309.

10. Itoh Y, Shinya K, Kiso M, Watanabe T, Sakoda Y, Hatta M, Muramoto Y, Tamura D, Sakai-Tagawa Y, Noda T, Sakabe S, Imai M, Hatta Y, Watanabe S, Li C, Yamada S, Fujii K, Murakami S, Imai $\mathrm{H}$, Kakugawa $\mathrm{S}$, Ito $\mathrm{M}$, Takano $\mathrm{R}$, Iwatsuki-Horimoto K, Shimojima M, Horimoto T, Goto H, Takahashi K, Makino A, Ishigaki H, Nakayama M, Okamatsu M, Takahashi K, Warshauer D, Shult PA, Saito R, Suzuki H, Furuta Y, Yamashita M, Mitamura K, Nakano K, Nakamura M, Brockman-Schneider R, Mitamura H, Yamazaki M, Sugaya N, Suresh M, Ozawa M, Neumann G, Gern J, Kida H, Ogasawara K, Kawaoka Y (2009) In vitro and in vivo characterization of new swine-origin H1N1 influenza viruses. Nature 460: 1021-1025.

11. Leung TWC, Tai ALS, Cheng PKC, Kong MSY, Lim W (2009) Detection of an oseltamivir-resistant pandemic influenza A/H1N1 virus in Hong Kong. J Clin Virol 46: 298299.

12. WHO, pandemic H1N1 2009 (2009). Update 107. http://www.who.int/csr/don/2010_07_02/en/index.html. Last accessed 27 February 2012.

13. Kamate SK, Agrawal A, Chaudhary H, Singh K, Mishra P, Asawa K (2009) Public knowledge, attitude and behavioural changes in an Indian population during the Influenza A (H1N1) outbreak. J Infect Dev Ctries 30: 7-14.

14. Leung GM, Ho L-M, Chen SKK, Ho S-Y, Bacon-Shone J, Choy RY, Hedley AJ, Lam TH, Fielding R (2005) Longitudinal assessment of community psychobehavioural responses during and after the 2003 outbreak of severe acute respiratory syndrome in Hong Kong. Clin Infect Dis 40: 1713-1720.

15. Cowling BJ, Ng DM, Ip DK, Liao Q, Lam WW, Wu JT, Lau JT, Griffiths SM, Fielding R (2010) Community psychological and behavioral responses through the first wave of the 2009 influenza $\mathrm{A}(\mathrm{H} 1 \mathrm{~N} 1)$ pandemic in Hong Kong. J Infect Dis 15: 867-876. 
16. May L, Katz R, Johnston L, Sanza M, Petinaux B (2010) Assessing physicians' in training attitudes and behaviors during the $2009 \mathrm{H} 1 \mathrm{~N} 1$ influenza season: a cross-sectional survey of medical students and residents in an urban academic setting. Influenza Other Respi Viruses 4: 267-275.

17. Carman WF, Elder AG, Wallace LA, et al (2000) Effects of influenza vaccination of health-care workers on mortality of elderly people in longterm care: a randomised controlled trial. Lancet 355: 93-97.

18. Dubé E, Gilca V, Sauvageau C, Boulianne N, Boucher F, Bettinger J, McNeil S, Gemmill I, Lavoie1 F, Ouakki M (2010) Canadian family physicians' and paediatricians' knowledge, attitudes and practices regarding $\mathrm{A}(\mathrm{H} 1 \mathrm{~N} 1)$ pandemic vaccine. BMC Research Notes 3: 102.

19. Kaboli F, Astrakianakis G, Li G, Guzman J, Naus M, Donovan T (2010) Influenza vaccination and intention to receive the pandemic H1N1 influenza vaccine among healthcare workers of British Columbia, Canada: a crosssectional study. Infect Control Hosp Epidemiol 31: 10171024.
20. Kamuhabwa AR, Jalal R (2011) Drug Use in Pregnancy: Knowledge of Drug Dispensers and Pregnant Women in Dar es Salaam, Tanzania. Ind J Pharmacol 43: 351-355.

21. Havlícková M, Pljusnin AZ, Tůmová B (1990) Influenza virus detection in clinical specimens. Acta Virol 34: 449-456.

\section{Corresponding author}

Dr. A. R. Kamuhabwa

PO Box 65013

School of Pharmacy

Muhimbili University of Health and Allied Sciences

Dar es Salaam, Tanzania.

Telephone: +255 757576985 (mobile), +255 222150748 (office)

Fax: +255 222150465

Email: akamuhabwa@muhas.ac.tz,apporug@yahoo.com

Conflict of interests: No conflict of interests is declared. 